\title{
AGNIESZKA FIRLEJ
}

Università Adam Mickiewicz, Poznań

\section{LA LETTERATURA PULP OSSIA GIOVANI CANNIBALI, IL NEONOIR, LA SCUOLA DEI DURI O IL GRUPPO 13? LE POLEMICHE SUI CONFINI DEL NUOVO GENERE LETTERARIO}

\begin{abstract}
Agnieszka Firlej, La letteratura "pulp" ossia "Giovani cannibali", il "Neonoir", la "Scuola dei Duri" o il "Gruppo 13"? Le polemiche sui confini del nuovo genere letterario [Pulp literature, i.e. Young cannibals, the Neonoir, the School of the Tough guys or Group 13? Controversies about frontiers of a new literary genre]. Studia Romanica Posnaniensia, Adam Mickiewicz University Press, Poznań, vol. XXXVII/1: 2010, pp. 85-98. ISBN 978-83-232-2145-6. ISSN 0137-2475. DOI 10.2478/v10123-010-0007-1.
\end{abstract}

The paper aims to present pulp as one of the main genres of contemporary Italian literature. This extreme kind of noir was born in the 1990s and immediately gained popularity among readers and caused controversy amid critics: from high and flattering opinions to complete rejection. The author examines the origin of the genre and the story of its development in Italy. She also describes the distinctive qualities of this kind of literature such as dark topics, eclectic form and mixture of languages. In addition, the paper shows various visions of pulp represented by different groups of authors: a serious and uncompromising or a light and amusing way of depicting the violence, inherited from Quentin Tarantino.

Gli anni Novanta del Ventesimo secolo costituiscono una vera svolta nella letteratura italiana in quanto per la prima volta nascono dei testi estremamente violenti e aggressivi, che differiscono da tutto quello che era stato scritto fino ad allora sulla penisola, e cambieranno assolutamente il panorama letterario. Le opere che rinnovano il campo letterario italiano vengono composte da autori che, evidentemente affascinati dalla crudeltà e prepotenza, si dilettano a descrivere minuziosamente omicidi, sporcizia e patologie. Il pulp, perché è questa la corrente di cui si tratta, è il genere che costituisce una delle più recenti manifestazioni della moderna letteratura noir, che la arricchisce sia del nuovo tipo di violenza che della nuova forma e del rinnovato linguaggio, perché gli strumenti utilizzati prima risultano insufficienti.

\section{IL TERMINE}

La questione rilevante è l'origine e il significato del nome pulp. Esso è soltanto uno tra diverse voci usate per descrivere l'insieme degli elementi letterari, perché comunemente si assiste ad un gran proliferare di varie etichette che designano lo stesso 
fenomeno. Pulp viene dalla lingua inglese e significa «pappa», ossia «un mucchio di materia organica vischiosa, umida, soffice...» (Sinibaldi, 1997: p. 39). Però la sua definizione nel contesto letterario sembra incerta perché si richiamano varie etimologie e riferimenti. Prima di tutto è un vocabolo utilizzato da due precedenti illustri. Il primo fu Charles Bukowski, scrittore americano, che intitolò il suo libro appunto Pulp. La sua è un'opera in cui vengono presentati i principi dello scrivere in maniera pulp, che l'autore realizza infilando in una storia d'avventura elementi caratteristici per i filoni letterari come giallo, fantascienza e romanzo psicologico, tutto questo raccontato dal protagonista principale con grande perspicacia e ironia con il tono volgare e grossolano. Il secondo padre della corrente a cui attinge il nome è Quentin Tarantino, il regista del famoso Pulp fiction. Nelle opere dell'artista americano, perché non solo nella pellicola da cui proviene il nome in maniera diretta, troviamo una miscela di orrore e di lievità, di tragico e di comico, tutti gli elementi poi reperibili nelle scritture di autori pulp, soprattutto quelli detti Giovani cannibali.

Comunque per il nome pulp si può trovare un'altra sua spiegazione ancora: in riferimento alla storia del genere, esso allude ai cosiddetti pulp magazines - una serie di pubblicazioni popolari di rapido consumo, con contenuti facili e stereotipati e cattiva qualità di carta. In questo senso il pulp moderno rispecchia chiaramente la similitudine tra il tipo di carta e la polpa, cioè la pasta lignea dalla quale proviene e si riferisce alle origini del filone.

Tuttavia la scelta del termine principalmente usato non è stata chiara, perché negli anni Novanta del Ventesimo secolo nascono tante altre denominazioni per il gruppo degli stessi elementi letterari. Alcuni critici per descrivere il genere utilizzano il termine splatter - nome che richiama gli schizzi di sangue e il gusto per il raccapricciante (letteralmente significa «il getto»). Tra tanti ricorrenti esiste anche la denominazione splatterpunk dove la parte aggiunta punk fa pensare all' antagonismo radicale. Comunque il genere nero e i suoi esponenti, oltre a quella del pulp e splatter guadagnano tante altre etichette che tentano di ridefinirli, tra le quali si trovano scrittori dell'horror, Cannibali, cattivisti, orroristi o noiristi. La letteratura stessa viene chiamata pure thriller, romanzi della paura, letteratura nera, neogotica oppure neonoir. La denominazione più ampia ovvero «nero», esistente già prima, si collega nell'immaginario degli italiani con l'idea di «eccesso di violenza» (Mondello 2004, p. 3) che si può trovare su uno o più livelli della letteratura: autore, canale, testo, codice, pubblico, contesto. Esistono anche nomi che si riferiscono ai tratti distintivi più particolari del pulp, come ad esempio il genere metropolitano per il fatto che le storie vengono ambientate prevalentemente nelle grandi città viste così come abituali luoghi di conflitti sociali. Invece Marino Sinibaldi presenta una metafora presa in prestito dal campo musicale che rispecchia la forza innovativa del filone. Egli ritiene che la letteratura pulp a paragone della letteratura tradizionale sia come Tecno e Hip Hop nei confronti della musica Rock e Pop - le nuove varianti musicali 
hanno anche prodotto una ininterrotta accelerazione della frase musicale delle vecchie forme. Per questo motivo tra le varie denominazioni del fenomeno pulp esiste anche speed.

\section{CARATTERISTICHE DEL GENERE}

Visto che già il termine stesso utilizzato per designare la corrente provoca una certa confusione, occorre assolutamente rintracciare l'insieme degli elementi tipici che permettono di specificare l'ondata. Da altri filoni contemporanei il pulp si distingue innanzitutto in due maniere: al livello tematico e strutturale. Il soggetto che forma il suo punto nodale è la presentazione di storie terribili ed esasperanti. È l'ondata che provoca brividi e ripugnanza attraverso la descrizione di schizzi di sangue e di atti atroci di violenza. La prepotenza che domina il mondo pulp gronda di crudeltà e spaventa con la visibile mancanza di moralità, in quanto sia spesso priva di alcun motivo. I rappresentanti del filone raccontano puro orrore, sono «testimonianza ieratica del male» (Trevi, 2006, p. 205), siccome in maniera evidente si fermano sui frammenti orrendi e trovano un vero piacere nella loro creazione. Gli autori pulp mettono in rilievo la loro lampante predilezione dell'orrore trattando spesso le atrocità alla leggera e abolendo i tabù. Giulio Ferroni nell'opera Dopo la fine. Sulla condizione postuma della letteratura, ritiene che questo approccio riduca la violenza ad una semplice apparenza, e parallelamente essa fa perdere il senso della sua influenza concreta sulla vita, poiché si sposta sul piano immaginario. A differenza del passato, non c'è più lotta contro lo stato o la classe sociale, la battaglia è diventata lotta per il puro piacere di lottare, perché si descrive l'aggressione senza alcuno sfondo psicologico, lasciando ogni analisi morale ed etica ai suoi lettori. E infine non ci propongono nessun lieto fine e non si accettano i confini tra Male e Bene, così la letteratura programmaticamente rifiuta il cosiddetto «effetto sociale positivo» (Mondello, 2004, p. 3). Come illustrazione di questa idea ci può servire il protagonista del racconto Il bagnoschiuma della raccolta Superwoobinda di Aldo Nove, che uccide e profana le salme dei suoi genitori senza nessun motivo profondo, soltanto perché loro non vogliono usare la stessa marca del bagnoschiuma che il loro figlio.

Come si è già menzionato, oltre agli elementi del mondo presentato, la letteratura pulp porta anche tratti caratteristici formali che la distinguono da altri generi letterari: è quindi una corrente amorfa, in essa si intrecciano tanti filoni che spesso sembrano contrastanti tra di loro, tali: noir, giallo, fantascienza, fumetto, thriller e horror, ma diversi autori attingono ad un repertorio ancora più ampio: romanzo rosa, psicologico, quello d'avventura e anche della fiaba. A prima vista pulp indica soltanto una produzione culturale che utilizza o ricicla materiali "bassi", ma in realtà esso attinge persino allo stile elevato e alla letteratura classica fondendo e mischiandoli insieme agli elementi della cultura popolare e di massa. 
Per quanto riguarda il livello linguistico, anche sotto questo aspetto non c'è l'uniformità. Si esprime attraverso diversi registri e stili, nutrendosi della letteratura tradizionale e giocando con i linguaggi tipici della pubblicità, televisione e cinema. Inoltre gli scrittori arricchiscono la loro produzione artistica con vari forestierismi, numerose parolacce e un'ampia gamma di neologismi. Secondo Daniele Brolli, è «una lingua ancora in via di formazione che raccoglie senza falsi pudori le sue parole dai palinsesti televisivi, dalla cultura di strada, dal cinema di genere, dalla musica pop» (Brolli, 2006, p. VIII). Invece Emanuele Trevi le chiama «parole-spazzatura», perché la lingua pulp non cerca di imitare una sintassi o un dizionario astratti (Trevi, 2006, p. 209).

La loro scrittura è vorace e, inesausta, fagocita tutto, inghiotte pure se stessa. Ne risulta un corpo narrativo squassato, che si diffonde attraverso gli squarci, pronto a geminare nuove narrazioni prive di strutture costrittive. È un linguaggio che si spinge costantemente oltre e che in questo "andare oltre" si libera del passato scoprendo nuovi territori che fanno piazza pulita degli ultimi avanzi di "letteratura" (Brolli, 2006, p. IX).

L'amalgama stilistico, linguistico e tematico serve innanzitutto al divertimento che però a sua volta non toglie alla letteratura pulp un'inconfutabile importanza nel campo letterario, poiché ne risulta «una scrittura laboratorio che mescola sostanze tra loro distanti, quasi seguendo la non lezione della "letteratura possibile": goliarda scolastica, slogan pubblicitari, melodie popolari, prodotti di consumo... il tutto impastato spesso con molto, molto sangue» (Trevi, 2006, p. VIII). Ad esempio Daniele Luttazzi, l'autore del racconto Cappuccetto splatter dell'antologia Gioventù cannibale, mescola insieme la favola tradizionale con elementi della quotidianità, tra i quali marchi di prodotti di consumo, tanta violenza e volgarità: il suo Cappuccetto è una modella che viene uccisa e consumata da un P.R., e infine ci aggiunge caratteristiche di fantascienza, perché la ragazza viene salvata dallo stomaco del malfattore dopo essere già stata divorata.

\section{ORIGINI DEL PULP ITALIANO}

Come si è già detto, il termine inglese pulp significa «polpa di legno» e dal punto di vista storico si riferiva a tante riviste, i soprammenzionati pulp magazines, caratterizzati dalle copertine colorate con immagini accattivanti, che apparvero in America nei primi decenni del Novecento. Dunque dovettero il nome alla scarsa qualità di carta utilizzata per la loro pubblicazione. In Italia comunemente si è convinti che il pulp arriva alla penisola soltanto nel 1996 con l'opera cinematografica Pulp fiction di Quentin Tarantino, invece non è del tutto vero. È giusto solo per quanto riguarda il nome che si diffonde solo dopo quell'anno, ma decisamente sbagliato per quanto riguarda la corrente in genere, perché alcuni elementi tipici della stilistica pulp appaiono già decine di anni prima. Certi tratti caratteristici si possono ritrovare nella prosa degli autori ap- 
partenenti alla tradizione letteraria come Dino Buzzati, Leonardo Sciascia, Giovanni Papini o Carlo Emilio Gadda, però la corrente stessa si presenta anche in maniera più evidente. Già nel dopoguerra a partire dal 1955 si pubblicano romanzi e fascicoli destinati al pubblico di massa e piuttosto sottovalutate dalla critica, e per questo motivo vendute spesso in edicola. Da questa condizione risulta che il genere letterario pulp si intreccia inseparabilmente per tutta la sua storia con il fumetto nero pure sottovalutato e spinto in vendita in chiosco. Tuttavia va ricordato che il pulp italiano deve molto non solamente al fumetto, ma pure alla fantascienza, con cui spesso viene scambiato, ne possono testimoniare le riviste Gamma e Galassia. Dagli anni Settanta in poi oltre ai generi soprannominati il pulp italiano più commerciale si gemella pure con l'erotico dando vita al cosiddetto porno-noir de I Neri del Momento.

In quel periodo il centro editoriale è costituito innanzitutto dalla città di Roma in cui tra l'altro la casa editrice E.P.I. (trasformata poi in E.R.P.) pubblica collane intitolate I Narratori Americani del Brivido, FBI - Gialli dello Schedario, Gli Assi Americani del Giallo, I Racconti di Dracula, Gli Esploratori dello Spazio, Far West, Prima Linea e così via. Ci opera la casa editrice G.E.I. che si occupa da una parte dei romanzi neri, come I Romanzi Diabolici o I Super Gialli Usa, dalla parte opposta invece di quelli rosa. La terza casa editrice romana KKK lancia I Classici dell'Orrore, Gialli Usa, I Gialli dell'Ossessione e Racconti di Guerra. Nascono così centinaia di collane che mescolano i generi popolari quali giallo, noir, spionaggio, horror, fantascienza, storie di guerra, western, romanzo rosa e d'avventura. Oltre alla miscela stilistica, tutte queste edizioni portano una caratteristica principale ossia quasi tutti i testi indicano falsamente americani come autori, e in realtà essi vengono composti nella maggior parte dei casi da scrittori italiani nascosti soltanto sotto pseudonimi suggerenti provenienza straniera - come esempio possono servire i nomi di Max Dave e Red Schneider. Dietro questi nomi falsi si nascondono soprattutto psichiatri, magistrati oppure gente del cinema.

Contemporaneamente a Milano si sviluppa il fumetto nero con l'iniziativa delle sorelle Giussani che lanciano Diabolik e l'editoriale di Max Bunker che pubblica tra l'altro Kriminal, Satanik, Gesebel, Agente SS018. Sempre nella città lombarda la casa editrice di Segrate inizia una forma più raffinata perché meno alternativa del pulp sotto la forma di due collane Segretissimo e $i$ Gialli Mondadori dirette da Laura Grimaldi e Marco Troppa.

Alla base dello sviluppo del pulp oggi possiamo individuare anche elementi negativi della storia, come ad esempio gli anni Settanta, i cosiddetti «anni di piombo», ossia il periodo della stasi letteraria e intellettuale. In quel tempo si pubblicavano pochi romanzi e gli intellettuali si occupavano innanzitutto della saggistica. Perciò già negli anni Ottanta del Ventesimo secolo la casa editrice Einaudi ha dedicato la serie Under 25 agli autori esordienti, tra i quali si sono trovati Stefano Benni, Aldo Busi oppure Andrea De Carlo, tutti loro scoperti da Pier Vittorio Tondelli. Lo stesso Tondelli attualizza I Ragazzi di vita di Pier Paolo Pasolini in Altri libertini nel 1980. 
Però per quanto riguarda il vero pulp, come ho già menzionato, la grande editoria ha sempre rifiutato di promuoverlo. Un'eccezione costituisce negli anni Ottanta Mondadori che pubblica un'antologia e un'intera collana con lo stesso titolo - Nero italiano. Sono progetti dedicati ai gialli e neri metropolitani composti dagli scrittori del Belpaese. Vale la pena di menzionare l'attività ancora di due personaggi che, a prescindere dalla tendenza generale di creare la letteratura nera soltanto a forma del poliziesco o nero «classici», si nutrono già della letteratura americana e propongono già un modello testuale diverso. Sono gli scrittori nascosti sotto pseudonimi: il primo è Alan D. Altrieri, detto anche "Chandler all'italiana», l'autore delle opere seguenti: Città oscura, Ultima luce e Kondor - testi che attingono al thriller e al noir. Il secondo precursore del pulp sta dietro il nome falso di Marc Saudade, è in realtà si tratta di Furio Colombo. Egli compone Bersagli mobili, L'ambasciatore di Panama, El Centro, Trappola a Hong Kong, fingendo inizialmente che fossero soltanto delle traduzioni della letteratura straniera: spagnola e inglese. A parte ciò in quel momento si dimostrano solo pochi movimenti alternativi e innovativi di poca portata.

\section{LA CORRENTE OGGI}

Finalmente arrivano gli anni Novanta colpiti dal cosiddetto «voltar pagina» rispetto al passato, ossia la letteratura comincia ad essere vitale, policentrica e ad interessarsi alla realtà contemporanea. L'anno 1996 è il periodo di svolta in Italia: sulla penisola giunge la famosa pellicola di Quentin Tarantino e in conseguenza Alessandro Baricco organizza un simposio dedicato al tema «Narrare dopo Pulp fiction» visto che il film costituisce una sorta di Iliade del nostro tempo e che l'opera traccia la trasformazione della narrazione, delle modalità del racconto (La Porta, 1999, p. 261-262). Ci si riflette sul fenomeno Tarantino in Italia e i suoi innumerevoli imitatori. Importanti sembrano persino i suoi precedenti film ossia Le iene (Reservoir Dogs) e anzitutto Natural Born Killers, in cui appaiono già elementi caratteristici per la sua estetica: violenza e amoralità. Tarantino si esprime con una specie di iperrealismo, ossia un realismo fuori misura che sembra trapassare nel suo contrario e diventa contemporaneamente una parodia e il suo stile viene caratterizzato con il termine «estetica della violenza» (Sinibaldi, 1997, p. 41). Come scrive Marino Sinibaldi, Tarantino nelle sue opere in maniera spettacolare esemplifica la decadenza della cultura di massa inserendo elementi della cultura alta e bassa, autentico e falso e costruendo pellicole postmoderne sia sul piano del linguaggio che del contenuto.

Nell'ultimo decennio del Ventesimo secolo si osserva il crescente interesse del genere pulp da parte della piccola, media e infine pure grande editoria e in effetti varie case editrici lanciano sul mercato nuove collane che si occupano della letteratura nera: tra l'altro da Einaudi c'è Vertigo e la recente Stile libero, di seguito Neonoir (Il Minotauro), Il nero italiano (Theoria), Noir (E/O), Vox-Noir (DeriveApprodi), I neri 
(Addictions) e Black is black di Fanucci. Essa si sviluppa anche grazie alla vertiginosa nascita di diverse riviste, sia quelle cartacee che esistenti soltanto su Internet, tra le quali si possono nominare: Mucchio Selvaggio, Experiment, Profondo Rosso, Container, Lamette, mammamiaquantosangue.

\section{GIOVANI CANNIBALI}

La letteratura pulp in Italia d'oggi diventa famosa a causa dell'attività del gruppo dei giovani scrittori che ispirandosi al Tarantino irrompono sulla scena letteraria nel famoso 1996 con «la prima antologia italiana dell'orrore estremo» (Brolli, 2006, copertina) intitolata Gioventù cannibale, volume a cura di Daniele Brolli che dà spazio a autori come Niccolò Ammaniti, Aldo Nove, Luisa Brancaccio, Alda Teodorani, Daniele Luttazzi, Andrea G. Pinketts, Massimiliano Governi, Matteo Curtoni, Matteo Galiazzo, Stefano Massaron e Paolo Caredda. I letterati sono «undici sfrenati, intemperanti, cavalieri dell'Apocalisse» (Brolli, 2006, copertina), scrittori che costituiscono una pura innovazione nel campo umanistico. Vengono presentati come «feroce e allegra brigata» (Brolli, 2006, copertina) che prende posizione di fronte alla peste, all'epidemia, ossia di fronte alla «patologia» del mondo e della società odierni, dello sfruttamento e del profitto di grandi metropoli. Il volume Gioventù cannibale nell'idea di Severino Cesari e Daniele Brolli era progettato per inaugurare la nuova collana Stile libero di Einaudi con cui volevano puntare piuttosto sulla forza ed energia spontanea dei testi. La prima raccolta inizialmente doveva intitolarsi Spaghetti splatter introducendo il termine splatter inteso come «schizzi di sangue», il che doveva rispecchiare le principali caratteristiche dei racconti. Il titolo finale invece, Gioventù cannibale, è apparso soltanto all'ultimo momento.

Il nome cannibale a sua volta è stato preso in prestito da una delle più note riviste di Andrea Pazienza del 1977 Cannibale. La denominazione stessa indica la loro energia gioiosa e provocante che traspare dai testi, rispecchia una scrittura che divora se stessa, che tende a fagocitarsi inglobando mondo narrato e mondo reale. Il nome deve anche all'ingoiamento di frammenti provenienti dalla tradizione alta e delle forme junk della cultura di massa, quindi divoramento di diversi linguaggi letterari. Nella loro produzione letteraria si comportano come veri e propri cannibali ingeriscono il passato e il presente, deglutiscono la cultura alta e bassa, come constata Linda Hutcheon. Però secondo la definizione del dizionario «cannibale» si riferisce semplicemente a «un uomo crudele e spietato» (Zingarelli, 2003, p. 295) che anche rispecchia l'atteggiamento dei protagonisti di tale genere.

Daniele Brolli nell'introduzione all'antologia Gioventù cannibale nel nome dei giovani autori rifiuta la funzione ideologica della letteratura e in conseguenza i Cannibali si presentano come nemici di ogni moralismo e della funzione militante della letteratura, e chiunque siano, cattivisti o buonisti, sono sempre politicamente indifferenti. 
Gli scrittori pulp, secondo il curatore, rinunciano alla retorica della fuga, si occupano piuttosto della descrizione de «le zone d'ombra della nostra vita quotidiana» (Brolli, 2006: p. VIII). Egli li definisce apologeti dell'orrore oppure apostoli del confuso.

Tutti i Cannibali utilizzano una lingua, anzi più lingue, «senza compromessi» (Brolli, 2006, p. X) con la quale vogliono abolire la cultura del moralismo e introdurre «una svolta dell'immaginario» (Brolli, 2006, p. X).

Ammaniti, Nove, Santacroce, Luttazzi: sono, questi, solo alcuni degli scrittori che, negli ultimi anni, hanno dato vita ad uno dei fenomeni letterari più originali e interessanti. Sia che si voglia chiamarli cannibali o definirli scrittori pulp il risultato non muta, in quanto le opere da loro prodotte hanno rappresentato, senza dubbio, una salutare novità in un panorama letterario nazionale spesso troppo stantio e legato ai canoni tradizionali (Chirumbolo, 2002, p. 312).

Il tratto distintivo dei Giovani cannibali che li differisce da altri gruppi che appartengono alla corrente pulp, è la comicità con la quale descrivono la bestialità (il fattore rifiutato intensamente dal gruppo del Neonoir). Marino Sinibaldi il loro atteggiamento vede come «civettare con l'orrore» (Sinibaldi, 1997, p. 65), perché descrivono le atrocità con onnipresente allegria. Il loro mondo quindi è sdoppiato, appeso «tra il disgusto e il riso, tra l'apocalisse e il carnevale» (Kristeva, 2006, p. 157). A che cosa gli serve però la comicità nella descrizione della violenza? Lo spiega di nuovo Marino Sinibaldi ponendo come l'esempio uno dei Cannibali, ma l'enunciato si può estendere anche sugli altri: «L'umorismo è per Ammaniti piuttosto "un modo per raccontare senza partecipazione"» (Sinibaldi, 1997, p. 63). Vuol dire che per mezzo della comicità si crea un distacco tra il racconto e il lettore che in questa maniera lo salva e protegge dalle atrocità a cui assiste. L'autore attraverso il riso incita il lettore a prendere distanza dall'azione atroce e crudele e lo aiuta a tornare intatto alla realtà.

I Giovani cannibali suscitano le opinioni dei critici molto controverse che frequentemente si contrappongono tra di esse. Lidia De Federicis li condanna per la negazione della storia, lo stile basso, i giochi linguistici, l'iperrealismo e per il loro atteggiamento altero. Invece Romano Luperini definisce i Cannibali una generazione di scrittori che non sa niente della letteratura e non ne vuole sapere nulla, perché è nata dopo la morte della letteratura. Nanni Balestrini li descrive come «narrative invaders anni 1960-1974» e rivolge l'attenzione sulla loro apertura sui linguaggi giovanili, il che è la visibile influenza del loro maestro Pier Vittorio Tondelli. Filippo La Porta, saggista e critico letterario, ritiene che i Cannibali siano poco innovativi dal punto di vista della trama, perché recuperano un gusto per narrare e ripetono per l'ennesima volta, seguendo la suggestione di Peter Bichstel, le storie già scritte creandone soltanto diverse varianti e complicazioni. Gli scrittori hanno senza dubbio la passione affabulatoria da story-teller dunque un debordante piacere di raccontare, ma contemporaneamente si ha la sensazione che potrebbero esprimersi con la stessa facilità attraverso altri mezzi di comunicazione - cinema, fumetto o pubblicità. Anche nella opinione del critico letterario Filippo La Porta sono un tipo di prodotto medio per il lettore medio che aspira alle tendenze di moda, comunque non si possono negare le ambiziose intenzioni dei 
Cannibali di descrivere la realtà contemporanea senza edulcorazioni e mutamenti, però egli lo vede piuttosto come «l'ennesimo travestimento della commedia all'italiana» (La Porta, 1999, p. 264). Marino Sinibaldi dopo aver letto dieci racconti della raccolta Gioventù cannibale, parla di pagine noiose, in cui gli autori presentano una continua gara all'effetto che raramente è sconvolgente. Solo alcuni scrittori di questo gruppo, come Ammaniti o Aldo Nove, sfuggono alla critica distruggente del saggista.

I Giovani cannibali vengono divisi in due sottocategorie: buonisti, cioè gli scrittori piuttosto moderati e tradizionalisti e cattivisti, autori assolutamente trasgressivi, comunque in genere la scrittura di tutti loro mira a un solo scopo: «[...] hanno scelto di infastidire, di raccontare al lettore esperienze sgradevoli, con personaggi antipatici o traditori» (Brolli, 2006, p. IX). Sono dicotomie da un lato completamente inutili secondo Marino Sinibaldi, dall'altro invece divisioni sbagliate nell'opinione di Fabio Giovannini e Antonio Tentori, autori dell'Introduzione all'antologia Cuore di pulp. Loro ritengono che i Cannibali cattivisti facciano finta di essere tali perché descrivendo le atrocità contemporaneamente imitano l'umorismo ultraviolento di Quentin Tarantino il che annienta la potenza della violenza descritta in maniera «seria». In effetti diventano consolatori altrettanto come i buonisti, perché dopo aver riso sul pericolo passa lo spavento.

E ritorna un vecchio limite della cultura nostrana: agli autori italiani non è concesso confrontarsi con i generi letterari, perché i generi sarebbero serie B, prodotti inferiori, spazzatura a paragone della Letteratura alta, quella con la L maiuscola. E così i finti pulp di oggi sono costretti alla risata obbligatoria, a dileggiare i generi in cui si imbattono per far contenti i critici e giornalisti snob, che non ammetterebbero mai nell'empireo degli autori un italiano che scrivesse «seriamente» storie horror, nere, splatter, ecc (Giovannini, Tentori, 1997, p. 9).

Giovannini e Tentori, parlando evidentemente anche nel nome degli autori che promuovono, preferiscono presentare la realtà così come è, utilizzando la chiave del realismo. Il primo li critica anche in altri testi per il loro atteggiamento conformista, tra cui in Storia del noir constata:

In realtà i Giovani cannibali hanno scelto la strada più comoda per strizzare l'occhio a editori, lettori e critici in cerca di sensazionalismo: la parodia dello splatter, dell'estremo, dell'ultraviolenza fatta di zombi e di descrizioni da voltastomaco, il tutto condito con «gergo giovanilistico». Ben poco a che fare con il noir (Giovannini, cit. da: Mondello, 2004, p. 13).

\section{IL NEONOIR}

Anche se alcuni critici considerano soltanto i Cannibali come rappresentanti del genere in Italia, sull'arena della letteratura nera c'è anche l'alternativa che si concentra intorno ai tre centri urbani: Roma, Milano e Bologna. Il primo gruppo, detto Neonoir, che si oppone acutamente alla stilistica dei Cannibali, è formato da diversi registi, scrittori e critici nell'estate del 1994 nella capitale dell'Italia. La loro azione prende 
l'avvio con una serie di incontri con Dario Argento e dà vita a un programma radiofonico di Radio Città Aperta Appuntamenti in Nero e uno spettacolo teatrale Il vampiro di Londra. Il termine neonoir per la prima volta è stato utilizzato nel 1991 da Maitland McDonagh nel contesto dello stile delle pellicole di Dario Argento. I neonoiristi riprendono dunque il nome deliberatamente, siccome assumono molti elementi della sua opera. Tra i suoi esponenti si trovano menzionati già Antonio Tentori e Fabio Giovannini, curatori e portavoce, inoltre ci sono Pino Blastone, Sabrina Delizia, Paolo De Pasquali, Nicola Lombardi, Marco Minicangeli, Aldo Musci, Claudio Pellegrini e, stranamente vista la posizione contraddittoria del gruppo verso i Cannibali, Alda Teodorani che pubblica pure nella raccolta Gioventù cannibale. Da soli gli autori si definiscono «un movimento-non movimento» e insieme lanciano varie iniziative come Banca degli inediti ossia la base informatica dei testi appartenenti al genere pulp per metterli a disposizione di tutti quelli che li vogliono leggere. I letterati collaborano con diversi piccoli editori tra cui Datanews, Stampa Alternativa, I libri dell'Altritalia/ Avvenimenti. Compongono insieme testi narrativi, radiofonici e teatrali e editano varie antologie e raccolte nelle introduzioni delle quali espongono dichiarazioni programmatiche e critiche allargando vivamente la discussione sul genere pulp in Italia. Ci possiamo trovare titoli di antologie quali: Neonoir, 16 storie e un sogno pubblicato nel 1994, Neonoir. Deliziosi raccontini con il morto dello stesso anno, Giorni Violenti. Racconti e visioni neonoir proveniente dal 1995, e le raccolte dell'inizio del Ventunesimo secolo: Bambini Assassini (2000), Grande macello. Racconti di horror estremo (2001), L'orrore della guerra. Racconti estremi di autori italiani (2003). Tra i volumi c'è anche il già menzionato Cuore di pulp. Antologia di racconti italiani pubblicato nel 1997, che presenta tredici racconti di giovani scrittori e ricorda due testi degli anni Settanta per rievocare il passato illustre del filone in Italia. Le storie raccolte sono prive di qualsiasi sguardo comico che concederebbe al lettore un qualunque tentativo di prenderne distanza. Tra gli autori di questa singola antologia si trovano Luigi Seviroli, il curatore - Antonio Tentori, Antonio Pedicini, Paolo De Pasquali, Ivo Scanner alias Fabio Giovannini, Sabrina Delizia, Claudia Camarca, Stefano Di Marino, Marco Minicangeli, Marco Scaldini, Nicola Lombardi, Aldo Musci e Alda Teodorani il racconto della quale apre l'antologia. Ciascuno di neonoiristi segue un percorso individuale, e così ad esempio Ivo Scanner privilegia la rivisitazione in chiave pulp della spy-story. Alda Teodorani invece, la cosiddetta «dark lady del gruppo» (Mondello, 2004, p. 10), alla quale appartiene il merito di aver scritto i testi più violenti e popolari, presenta innanzitutto, il «punto di vista di Caino» (Mondello, 2004, p. 10), ossia la prospettiva dell'assassino. Un ottimo esempio costituisce il racconto $E$ Roma piange della raccolta Gioventù cannibale che presenta l'ottica del sicario, «spazzino», come lo si chiama nel testo, assunto da un signore anziano malato di cancro, che non vuole più guardare per strada la gente povera che pulisce i vetri o vende gli accendini.

Fabio Giovannini che opera anche molto attivamente sul campo critico, formula un elenco di quattro elementi che costituiscono la base della produzione del Neonoir. 
Come primo punto enumera la figura dell'assassino che occupa la posizione centrale nella narrazione. Di seguito si parla della struttura dei testi ovvero il voler mescolare diversi generi tra i quali noir, giallo, spy story, l'horror e cyber usando il criterio multimediale, inserendoci cinema, televisione e fumetto. Il terzo principio secondo Giovannini è la collocazione delle storie tra cronaca nera e immaginario, in quanto la realtà costituisca il punto di partenza e la fantasia, l'elemento che serve a oltrepassarla. L'ultima condizione è il privilegio delle situazioni estreme che rifiutano ogni perbenismo. Parallelamente il Neonoir, contrariamente ai Giovani Cannibali che rifiutano ogni posizione militante, si attribuisce una funzione politico-ideologica. Claudio Pellegrini nell'Introduzione al volume del 1997 Italian tabloid. Crimini e misfatti dentro il cuore dello Stato dichiara:

Con il «noir» il romanzo ha la possibilità di tornare all'impegno civile, alla lotta politica e culturale, alle scelte scomode, al coraggio di essere autori ed interpreti.

Il «noir» può di nuovo alzare le barricate culturali ed umane, può consentirci di portare la Fantasia, se non al potere, almeno all'opposizione. (Pellegrini, cit. da: Mondello, 2004, p. 14)

La stessa opinione dimostra Giovannini dicendo che il vero noir dovrebbe portare guai ai suoi autori, perché è maledetto e dà fastidio alle autorità.

Il gruppo Neonoir polemizza non soltanto con i Cannibali, ma anche con il Gruppo 13, vicino al nero e poliziesco nelle loro forme tradizionali. Anzi, i neonoiristi vogliono superare la tradizione letteraria al livello linguistico, contestuale, della costruzione delle trame e della narrazione.

Comunque nella capitale del Belpaese si svolge almeno un'altra impresa interessante legata alla corrente noir. All'università degli Studi di Roma La Sapienza, intorno alla cattedra di Sociologia della Letteratura e di Letteratura italiana moderna e contemporanea dal 2004 si organizza un progetto intitolato Roma noir che, oltre agli incontri annui con vari scrittori, bandisce un concorso per il migliore racconto che in seguito viene pubblicato on line. Così, Elisabetta Mondello, studiosa romana che si occupa delle problematiche del filone, promuove nuovi autori e aiuta la corrente a svilupparsi. Nel 2009 tra le storie premiate si è trovato un racconto di Andrea Franco Ildemone della Buonanotte in cui il protagonista principale descrive la sua ossessione di una certa Silvia, si assiste alla scena del coito molto sensuale tra loro due e nella scena finale, scopriamo con disgusto che la ragazza è la figlia del personaggio, il che cambia pienamente il significato del testo: con una sola frase lo catapulta dalla storia d'amore direttamente al genere noir.

\section{ALTRI CENTRI DELLA LETTERATURA NOIR}

La seconda città in cui si sviluppa la corrente nera in Italia è Bologna e la presenza del cosiddetto Gruppo 13, che i critici iscrivono piuttosto al genere noir. La sua storia inizia nel 1990 e tra i suoi fondatori si trovano Massimo Carloni, Pino Cacucci, 
Nicola Ciccoli, Danila Comasti Montanari, Marcello Fois, Loriano Macchiavelli, Loris Marzaduri, Gianni Materazzo, Sandro Toni e il suo esponente più celebre, Carlo Lucarelli, l'autore tra l'altro dei libri Almost Blue (1997), Il giorno del lupo (1998), Lupo mannaro (2001) e Il lato sinistro del cuore (2003). Gli scrittori pubblicano sia testi individuali che raccolte e antologie di più autori. Tra i volumi collettivi ci sono: I delitti del gruppo 13 del 1992, Giallo, nero e mistero del 1995, Delitti sotto l'albero edito nel 1999 e Capodanno nero pubblicato nel 2000.

Oltre all'attività del Gruppo 13, la zona bolognese ha il merito di creare una vera e propria tradizione narrativa gialla che si definisce una specie di «caso Emilia Romagna». Ė il merito anche di scrittori quali Eraldo Baldini, l'autore di diversi romanzi tra cui Bambine del 1995, Daniele Brolli, letterato appeso tra horror e noir, fra l'altro il curatore di Gioventù cannibale e Luigi Bernardi, scrittore e saggista che insieme a Carlo Lucarelli conduce la collana dedicata all'innovazione letteraria Stile libero di Einaudi.

L'ultimo centro italiano nel quale si sviluppa la letteratura noir è la città di Milano. Intorno ad Andrea G. Pinketts, «scrittore noir da bar, marciapiedi e boulevards» (Mondello, 2004, p. 8) come si autodefinisce l'autore, si forma la cosiddetta Scuola dei Duri. La figura di Pinketts è difficoltosa da definire visto che il suo stile è basato fortemente sulla grottesca e surrealismo e per questo motivo viene spesso inserito nella letteratura umoristica anziché all'interno pulp. Esemplare per il suo stile difficilmente descrivibile e definibile è il frammento iniziale del romanzo L'assenza dell'assenzio: «Mi svegliai con il viso sprofondato nel pelo pubico di una sconosciuta. Avevo trentacinque anni. Come dire: "Nel mezzo del cammin di nostra vita / mi ritrovai per una selva oscura..."» (Pinketts, 2006, p. 11). Comunque pubblica pure nella raccolta Gioventù cannibale, non prescinde dalla violenza e crudeltà tipiche per il pulp, dunque è appeso tra diversi filoni e tendenze anche dentro la casella della letteratura nera.

L'antologia che costituisce contemporaneamente il manifesto della scuola proviene dal 1995 ed è intitolato Crimine Milano giallo-nera. Raccolta di inediti della Scuola dei Duri. A parte il personaggio centrale di Andrea G. Pinketts, ci editano i testi Gaetano Cappelli, Andrea Carlo Cappi, Alessandro Riva e Lorenzo Viganò.

Tuttavia in diverse opere critiche incontriamo varie altre divisioni degli autori pulp o noir in gruppi e sottogruppi ancora diversi. Ad esempio Renato Barilli descrive il caso della «vitamina SS9» (Barilli, 2000, p. 121), ossia accumula insieme Isabella Santacroce, Tiziano Scarpa e Aldo Nove per il comune «stilismo ardito e baldanzoso, sicuro e radicale anche se applicate per vie tra loro assai distanti» (Barilli, 2000, p. 121). Per la simile tendenza «romanzesca allo stato puro» (Barilli, 2000, p. 126) Niccolò Ammaniti, conosciuto per la costruzione raffinata delle trame, è accompagnato da Matteo Galiazzo e Stefano Massaron, il più tradizionale tra i Giovani cannibali in quanto utilizzi una storia lineare, condotta attraverso la narrazione classica e scritta in linguaggio quasi privo di volgarità, si trova accanto a Mauro Covacich visto che tutti e due dimostrano «il contrasto di fondo tra un'umanità vogliosa di praticare sentimenti 
normali, schietti, affabili, e il delinearsi, sullo sfondo, di orridi crimini che ledono ogni diritto e ogni speranza» (Barilli, 2000, p. 154). Il problema di qualificare gli autori noir a un solo gruppo letterario risulta dal fatto che ogni scrittore frequentemente porta caratteristiche diverse dagli altri membri del suo gruppo e succede che proprio quelle lo accostino ad un altro autore del gruppo concorrente. I letterati pulp dunque sono difficili da incasellare e richiudere una volta per tutte in una stretta etichetta. Sono personaggi a cui piacciono le nuove sfide intellettuali e artistiche, per questo una volta pubblicano nelle raccolte più legate al noir per poi editare racconti insieme agli scrittori di fantascienza, come ad esempio agisce volentieri Niccolò Ammaniti che è uno degli scrittori dei racconti sia in Gioventù cannibale, sia Crimini che presenta i testi piuttosto noir sia Tutti i denti del mostro sono perfetti - antologia delle storie di fantascienza.

Dunque com'è il genere pulp in Italia? Sviluppatosi negli anni Novanta, si è suddiviso in vari gruppi e scuole che gareggiano per la loro autonomia all'interno della corrente noir, tuttavia senza alcun dubbio hanno tanti punti in comune. L'Elemento che costituisce il perno del filone è la presenza continua di una estrema violenza e di una raccapricciante crudeltà in quanto tema principale, descritte anche frequentemente dal punto di vista del criminale, il che lega autori tanto diversi come Aldo Nove e Antonio Tentori. In seguito tutti gli esponenti della letteratura pulp, non importa come li chiamiamo, Cannibali, Neonoir, o in altra maniera ancora, si nutrono di diversi stili, registri, tradizioni e linguaggi, così l'amorfismo e il gioco continuo con la forma è diventato la loro ricchezza, che a sua volta complica ai critici il compito d'iscrizione delle opere a un solo genere letterario. Quello che distingue differenti autori e gruppi è innanzitutto la realizzazione del concetto pulp: i Cannibali ne parlano volentieri con la leggerezza e l'umorismo che allontana il brivido dello spavento, e invece il Neonoir compone i testi esclusivamente con rigida serietà. Li differisce anche l'idea della funzione della letteratura che si trova tra il puro divertimento, caratteristico per il primo gruppo, e il trasmettere di una certa ideologia che dichiarano i membri del secondo. Si potrebbe dire dunque che la lotta per dominare il pulp in Italia è nata tra gli scrittori stessi, che da un lato dimostrano più similitudini di quanto sono capaci di ammettere, dall'altro la diversificazione dimostra quanto vivamente si espande la letteratura nera nel Belpaese.

\section{BIBLIOGRAFIA}

Barilli, R. (2000). È arrivata la terza ondata. Torino: Testo \& Immagine s.r.l.

Brolli, D. (2006). Gioventù cannibale. Torino: Einaudi.

- (2006). Le favole cambiano. In D. Brolli (Eds.), Gioventù cannibale (pp. V-X). Torino: Einaudi.

Cesari, S. (2002). Les jeunes cannibales, Magazine littéraire (407), 20-24.

Chirumbolo, P. (2002). Italian Pulp fiction the New Narrative of the "Giovanni Cannibali" Writers, Rivista di studi italiani (2), 312-315. 
De Cataldo, G. (2005). Nota del curatore, Crimini (pp. V-VII).Torino: Einaudi.

Evangelisti, V. (1997). In difesa della fantascienza, Tutti i denti del mostro sono perfetti (pp. 5-13). Milano: Oskar Mondadori.

Ferroni, G. (1996). Dopo la fine. Sulla condizione postuma della letteratura. Torino: Einaudi.

Giovannini F. \& Tentori, A. (1997). Introduzione. Il vero pulp italiano. In F. Giovannini \& A. Tentori (Eds.), Cuore di pulp (pp. 3-10). Viterbo: Nuovi Equilibri.

Gola, S. \& Bastiaensen, M. (2004). Sguardo sulla lingua e la letteratura italiana all'inizio del terzo millennio. Firenze: Franco Cesati Editore.

Kristeva, J. (2006). Poteri dell orrore. Milano: Spirali.

La Porta, F. (1999). La nuova narrativa italiana. Travestimenti e stili di fine secolo. Torino: Bollati Boringhieri.

Lardo, C. \& Pierangeli, F. (1999). L'ultima letteratura italiana. Roma: Vecchiarelli Editore.

Mondello, E. (2005). Il Neonoir. Autori, editori, temi di un genere metropolitano. Retrieved June, 16, from http://romanoir.it/pdf/Mondello_Il Neonoir.pdf

- (2004). La narrativa italiana degli anni Novanta. Roma: Meltemi.

Nove, A. (1998). Superwoobinda. Torino: Einaudi.

Ottonieri, T. (2000). La Plastica della Lingua. Stili in fuga lungo una età postrema. Torino: Bollati Boringhieri.

Pinketts, A.G. (2006). L'assenza dell'assenzio. Milano: Oskar Mondadori.

Popper, K.R. (1994). Cattiva maestra televisione. Milano: Donzelli.

Rosa, A.A. (1999). Un altro novecento. Firenze: La nuova Italia.

Serkowska, H. (2002). "Kanibale" w prozie włoskiej schyłku wieku. In G. Gazda, A. Izdebska \& J. Płuciennik (Eds.), Wokół gotycyzmów: wyobraźnia, groza, okrucieństwo (pp. 251-258). Kraków: Universitas.

- (2006). Literatura włoska w toku. Wrocław: Zakład Narodowy im. Ossolińskich - Wydawnictwo.

Sinibaldi, M. (1997). Pulp. La letteratura nell'era della simultaneità. Roma: Donzelli.

Trevi, E. (2006). Spazzatura e violenza: sull estetica cannibale. In D. Brolli, Gioventù cannibale (pp. 203-209). Torino: Einaudi.

Zingarelli, N. (2003). Lo Zingarelli. Vocabolario della lingua italiana. Bologna: Zanichelli. 